\title{
A Framework to Evaluate the Quality Assurance Parameters of Fashion Technology Courses in India
}

\author{
Arul Kumaravelu ${ }^{1, *}$, ESM Suresh $^{2}$ \\ ${ }^{1}$ Department of Knitwear Design, National Institute of Fashion Technology, Chennai, India \\ ${ }^{2}$ Department of Civil Engineering, National Institute of Technical Teachers Training and Research, Chennai, India
}

Received October 20, 2020; Revised March 4, 2021; Accepted March 23, 2021

\section{Cite This Paper in the following Citation Styles}

(a): [1] Arul Kumaravelu, ESM Suresh, "A Framework to Evaluate the Quality Assurance Parameters of Fashion Technology Courses in India," Universal Journal of Educational Research, Vol. 9, No. 5, pp. 1025 - 1036, 2021. DOI: 10.13189/ujer.2021.090515.

(b): Arul Kumaravelu, ESM Suresh (2021). A Framework to Evaluate the Quality Assurance Parameters of Fashion Technology Courses in India. Universal Journal of Educational Research, 9(5), 1025 - 1036. DOI: 10.13189/ujer.2021.090515.

Copyright $\bigcirc 2021$ by authors, all rights reserved. Authors agree that this article remains permanently open access under the terms of the Creative Commons Attribution License 4.0 International License

\begin{abstract}
The focus of the article is the development of a Quality Assurance Framework for the institutions that offer Fashion Technology Courses in India. The present study explores and analyses the different quality frameworks and accreditation systems prevailing in the higher education system in India. Based on the extensive review of the past literature and the existing quality systems, a new set of quality assurance framework parameters has been indented and developed for assessing the quality of Fashion Technology Courses in India. The descriptive study was conducted with the faculty members of National Institute of Fashion Technology, India. The profound analysis identified the quality parameters applicable for various stakeholders like students, faculty, management, assessment and evaluation experts, curriculum designers etc. The key parameters used to develop the quality assurance framework are categorized as Resources, Education Management, Instructional Planning \& Delivery, Assessment \& Evaluation, Generic Skills and Learning Experiences and Skill Requirements. The study has revealed several implications on the various aspects contributing to these parameters and the ranking for their respective impact on ensuring quality in Fashion Technology education in India. A deeper understanding and focus to improve the top-rated contributors to these key parameters will ensure Enhanced Quality Framework for the Institutions offering Fashion technology Courses in India.
\end{abstract}

Keywords Quality Assurance, Fashion Courses,
Fashion Technology, Accreditation, Quality Framework

\section{Introduction}

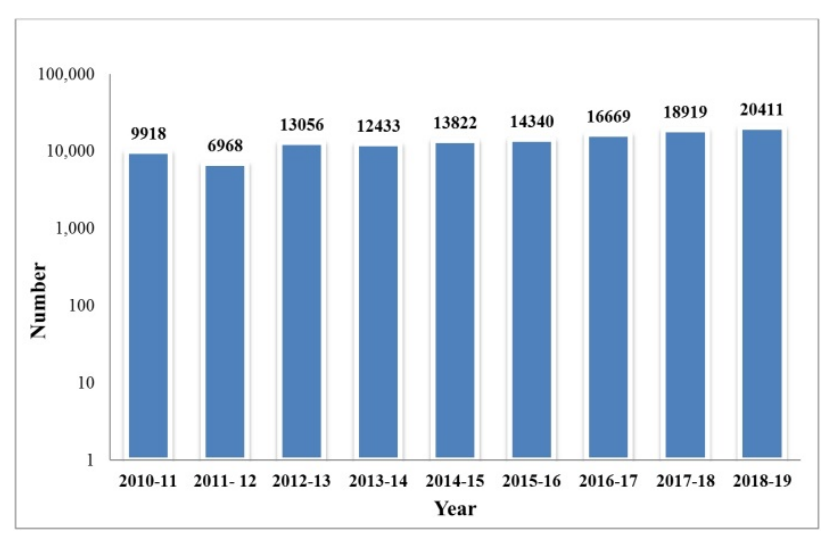

Figure 1. Enrolment of students in Fashion Technology Courses in India (AISHE, Report 2019)

Indian higher education is expanding and to exercise control over its movement the Central \& State Government has introduced various regulations and reforms like Technical Education Quality Improvement Program (TEQIP) (Saeki \& Imaizumi, 2013), (Stella \& Bhushan, 2011). The accreditation process has been set up which makes it compulsory for every educational institution to avail, accreditation under the University 
Grants Commission (UGC) (Mandatory Assessment and Accreditation of Higher Educational Institutions) Regulations 2012, to acquire the title of university. The accreditation is given for a period of 5 years by the accreditation agencies like the National Assessment and Accreditation Council (NAAC) and the National Board of Accreditation (NBA) (Dhanuraj \& Kumar, 2015). (Venkatesh, 2013) (Mishra, 2007) The National Assessment and Accreditation Council (NAAC) is responsible for addressing the issues related to deterioration in the quality of education and plays an active role in assessing the curricular, teaching-learning aspects, a research consultancy, infrastructure, student support, and management practices. It identifies the internal areas of planning and helps the educational institutes to initiate innovative techniques of teaching. (Van Zanten, Norcini, Boulet \& Simon, 2008); (Sakunth Kumar, 2018); (Parth Welankar, 2019); (Khan, 2019). On the other hand, the National Board of Accreditation (NBA) is responsible for accrediting college or universities offering Diploma, Undergraduate, and Postgraduate courses. The main aim of the association is to promote new $\&$ innovative methods of teaching, assure the quality of education, and provide feedback to bring improvements in the educational programmes (Sakunth Kumar, 2018). To cater the needs for industry, the number of institutions offering fashion technology courses has tremendously increased in the last few years. According to All India Survey on Higher Education (AISHE, 2019), gross enrolment of students in fashion technology courses has doubled in the last nine years (Figure 1). Thus, it is vitally important that the quality of the education must be ensured in the institutions that offer Fashion Technology Courses. To make focus on different quality assurance parameters and practices for fashion technology, the dimensions like curricular aspects, teaching-learning \& evaluation process; research consultancy and education; infrastructure and learning resources; student support and progression; governance, leadership, and management; innovative and best practices are taken into consideration. Teacher professional activities, involvement of students in research and practical activities enhances the quality of teaching and creativity of students. (Alena D. Matraeva \& Marina V. Rybakova, 2020). The curriculum defines the road map for the provision of quality education whereas resources indicate about the physical requirements like infrastructure, furniture, labs. The learner's profile describes the experiences, cultures, expectations, and motivations of the students, while teachers' learning is associated with knowledge, skills, and abilities of the faculty to impart learning to the students. Governance and student support are related to the following of the vision, team learning, and system thinking. All the parameters will help the learners to gain high quality of learning in ten fashion technology institutes (Pabrekar, Sharma, Kekare, and Barve, 2015) (Zaki \& Rashidi, 2013); (Dr.
Mohan Lal, 2017). The current research study which is related to the engineering education provides details about the quality assurance in higher education, success, challenges in quality assurance (QA) review practices, and quality assurance practices in fashion technology.

\section{Objective of the Research}

- To analyze different Quality Framework and Accreditation Systems prevailing in Higher Education system in India

- To identify different Quality Assurance Parameters adopted in Fashion education.

- To rank the Quality Assurance Parameters that have an impact on the quality of Fashion Education.

- To develop the Quality Assurance Framework for Fashion Technology Courses in India.

\section{Quality Assurance in Higher Education}

\subsection{Quality Assurance in Global Higher Education}

Ryan, (2015); Bordia, (2001) analysed that quality assurance in global higher education takes three factors like competitive, regional recognition, and international quality framework is taken into consideration. In India, the quality assurance is done by national assessment and accreditation council (NAAC) which acts an external quality assurance (EQA) agency. The agency is responsible for monitoring, protecting, and enhancing the quality of education in higher education so that quality outputs will be achieved effectively. For example, to maintain quality assurance in higher education in United States (US) regional accrediting bodies like accrediting bodies are Western Association of Schools and Colleges (WASC) Accrediting Commission for Senior Colleges and Universities have been established whereas, Quality Assurance Agency for Higher Education (QAA) is responsible for maintaining the quality of education in the United Kingdom. The Quality Matters Rubric have been adopted by Fudan University in China to improve the framework of quality assurance and provided effective professional development. It also develops a relationship between the funding, management and quality assurance in developing countries.

\subsection{Overview of Accreditation as a Mechanism to Ensure Quality in Higher Education}

As per Press Information Bureau Government of India, (2019); Verma, (2016) point of views, accreditation agencies play a major role in quality assurance by adopting the reviewing process of the functioning of the 
various higher educational institutions. The accreditation process acknowledges the transformative role of education, facilitates its expansion, provides information about the standards of the higher educational institutions, and helps the students to choose courses \& institutions as per grades. As a result, the gap in the quality of provision of quality of education is identified and necessary improvement is done to increase the institutional rankings leading to more research, innovation \& creation of greater learning eco-system.

\subsection{Models of QA to Ensure Quality in Higher Education}

According to Singh, (2001) to focus on the Quality assurance models, Baldrige criteria, ISO 9000-2000, Capability Maturity Model, Six Sigma and Total Quality Management are used. The Baldrige criteria model is based on the concept of the ever-improving value of the students, stakeholders and involves the organizational effectiveness \& capabilities towards the learning process. West, (2000) analysed that the International Organization for Standardization (ISO) 9000-2000 model is based on the process model that involves continuous improvement. It includes eight quality principles which are Customer focus, leadership, involvement of people, process approach, systems approach, continuous improvement, fact-based decision-making, and mutually beneficial supplier relationship. Duarte \& Martins, (2013) found that the Capability Maturity Model is based on managing intent development process and maintenance of procedures so that outcomes will be achieved appropriately. It helps in resource optimization and increases the responsiveness of the organizations. Al-Atiqi \& Deshpande, (2009, March) Six Sigma model is based on the usage of statistical tools like standard deviation to evaluate the deviations in the learning process. It improves quality by identifying the defects in the work. Mehta, Verma \& Seth, (2014) examined that the Delphi technique is applied through quality management (TQM) in most of the higher educational institutions to recognize the hierarchy of actions and enhance the quality of engineering education. The use of all these models helps in improving the quality of higher education. J. Bennedsen \& S. Rouvrais (2020) recommends international CDIO model framework of three guidelines: Programme goals, designing \& operative systems and self -assessment for quality assurance.

\subsection{Student Involvement with Quality Assurance}

Ryan, (2015) found that the involvement of students in quality assurance practices is necessary and is done by performing certain activities like responding to focus group interviews, questionnaires, and participating in quality assurance working groups. The association of students in quality assurance processes provides a good learning experience to students and provides learning insights to the provision of education which could not be provided by any other agency. It also helps to increase transparency, enhances the quality of higher education and creates a sense of ownership of the educational programs.

\section{Success and Challenges in QA Review Practices}

\subsection{Different Quality Assurance Practices Followed in Different Colleges of India Providing Courses in Fashion Technology}

As per Senthilkumar \& Arulraj, (2011); Dill, (2007) point of views, internal, and external quality assurance practices are followed by different colleges of India providing courses in fashion technology. For example, internal quality assurance practices are done by colleges like National Institute of Fashion Technology Mumbai, National Institute of Fashion Technology, Chennai, National Institute of Fashion Technology, Patna to monitor and enhance the quality of education provision in the organization. Additionally, the formation of Internal Quality Assurance Cell (IQAC) has been made compulsory in every college or university to increase the internal quality of education by conducting internal and external audits.

Gnanam \& Chairman, (2008) examined that external quality assurance practice like National level External Quality Assurance (NEQA) mechanism has been established by the fashion institutes like National Institute of Fashion Technology Hyderabad, Pearl Academy Delhi, National Institute of Fashion Technology Kolkata to ensure academic audit, assessment and accreditation is done by taking the international practices into consideration. Figure 1 shows the enrolment of students in fashion technology courses in India.

\section{Methodology}

Research methodology is the mixture of various research approaches and assumptions that help the researcher to collect reliable data (Creswell \& Creswell, 2017). The current study adopted both descriptive and analytical or both qualitative and quantitative research approach. The study also considered the mixed research paradigm to analyse different Quality Frameworks and Accreditation Systems prevailing in Higher Education. The following parameters and criteria are identified for the survey. 
Table 1. Quality Parameters for Fashion Technology Courses

\begin{tabular}{|c|l|}
\hline Sl. No & Quality Parameters \\
\hline 1 & $\begin{array}{l}\text { Resources } \\
\bullet \quad \text { Students } \\
\text { Faculty } \\
\text { Infrastructure }\end{array}$ \\
\hline 2 & Education Management \\
\hline 3 & Curriculum Content and Quality \\
\hline 4 & Assessment \& Evaluation \\
\hline 5 & Learning Outcomes \\
\hline 6 & Generic Skills and Learning experiences \\
\hline 7 & Skillset Requirement the Students \\
\hline
\end{tabular}

\subsection{Data Collection \& Analysis}

To develop quality assurance parameters for Fashion Technology Courses in India, data are to be collected through questionnaire and survey, and through secondary data analysis of journals, institutes websites, publications. The survey was conducted among the faculty members of National Institute of Fashion Technology, India. The total respondents for the survey using a questionnaire were 162 . The Statistical Analysis conducted by the using the SPSS tool.

\section{Results Discussion and Conclusion}

This section presents the results along with the conclusions generated based on the findings of the study which have conformed with the research goals established primarily in the research. The result and discussion segment emphasize the reactions and factual data accomplished while conducting the study assisting further the achievement of the objectives related to the study. The conclusions are drawn based on the results along with the additional literature referred to in the study and assessment with the existing body of literature evaluated is also discussed.

\subsection{Result and Discussion}

Considering the concern of the Indian higher education sector of addressing the problems related to the decline in the level of the quality of the educational courses, assessment through quality assurance has become a necessity. To concentrate on the diverse quality assurance parameters in the field of fashion technology education, multidimensional framework needs to be considered. The present study is an attempt to contribute to the existing literature by furnishing details regarding the significance of quality assurance in higher education in the context of fashion technology, challenges encountered in quality assurance review practices along with standard quality practices pursued in fashion technology. The study followed the quantitative as well as the qualitative approach and also followed the descriptive as well as analytical method in designing the research. Mixed data collection techniques facilitated in comparing the Quality Assurance Practices of Fashion Technology Courses in the context of Indian institutions and attempted to develop Quality Assurance Parameters for Fashion Technology Courses in India. Quantitative data were collected through a questionnaire by performing a survey with the sample size of 162 respondents comprising teachers at National Institute of Fashion Technology in India.

\subsection{Results from Analysis}

The results were inferred based on the questionnaire conducted by survey method. The various outcomes were deduced through thematic and statistical analysis. Among the respondents involved, and their percentage most of the respondents approximately $37.3 \%$ were between the ages of $41-45$ years and $26.5 \%$ between the age of above 50 . 
Table 2. Quality Parameters for Resources - Students

\begin{tabular}{|c|c|c|c|c|c|c|c|c|c|c|}
\hline $\begin{array}{c}\text { S. } \\
\text { nos. }\end{array}$ & Description & & $\begin{array}{l}\text { Strongly } \\
\text { Disagree }\end{array}$ & Disagree & $\begin{array}{c}\text { No } \\
\text { opinion } \\
\end{array}$ & Agree & $\begin{array}{c}\text { Strongly } \\
\text { Agree }\end{array}$ & Mean & $\begin{array}{c}\text { Std. } \\
\text { Deviation }\end{array}$ & Rank \\
\hline \multirow[b]{2}{*}{1} & \multirow{2}{*}{$\begin{array}{c}\text { Students } \\
\text { Admission } \\
\text { Policies and } \\
\text { regulation }\end{array}$} & Frequency & 3 & 10 & 30 & 36 & 83 & \multirow[b]{2}{*}{4.15} & \multirow[b]{2}{*}{1.047} & \multirow[b]{2}{*}{1} \\
\hline & & Percent & 1.9 & 6.2 & 18.5 & 22.2 & 51.2 & & & \\
\hline \multirow{2}{*}{2} & \multirow{2}{*}{$\begin{array}{l}\text { Student } \\
\text { Strength }\end{array}$} & Frequency & 0 & 19 & 33 & 77 & 33 & \multirow{2}{*}{3.77} & \multirow{2}{*}{0.909} & \multirow{2}{*}{5} \\
\hline & & Percent & 0 & 11.7 & 20.4 & 47.5 & 20.4 & & & \\
\hline \multirow[b]{2}{*}{3} & \multirow[b]{2}{*}{$\begin{array}{c}\text { Sufficient } \\
\text { Resources for } \\
\text { Academic } \\
\text { advice for } \\
\text { students } \\
\text { (Mentoring) }\end{array}$} & Frequency & 0 & 8 & 36 & 72 & 46 & \multirow[b]{2}{*}{3.96} & \multirow[b]{2}{*}{0.841} & \multirow[b]{2}{*}{2} \\
\hline & & Percent & 0 & 4.9 & 22.2 & 44.4 & 28.4 & & & \\
\hline \multirow[b]{2}{*}{4} & \multirow{2}{*}{$\begin{array}{c}\text { Students } \\
\text { Contribution to } \\
\text { cultural \& } \\
\text { Economic } \\
\text { development }\end{array}$} & Frequency & 0 & 16 & 34 & 60 & 52 & \multirow[b]{2}{*}{3.91} & \multirow[b]{2}{*}{0.961} & \multirow[b]{2}{*}{4} \\
\hline & & Percent & 0 & 9.9 & 21.0 & 37.0 & 32.1 & & & \\
\hline \multirow[b]{2}{*}{5} & \multirow{2}{*}{$\begin{array}{c}\text { Alumni } \\
\text { interaction \& } \\
\text { support }\end{array}$} & Frequency & 0 & 10 & 49 & 42 & 61 & \multirow{2}{*}{3.95} & \multirow{2}{*}{0.964} & \multirow{2}{*}{3} \\
\hline & & Percent & 0 & 6.2 & 30.2 & 25.9 & 37.7 & & & \\
\hline \multirow[b]{2}{*}{6} & \multirow{2}{*}{$\begin{array}{l}\text { Admission of } \\
\text { Students from } \\
\text { other countries }\end{array}$} & Frequency & 3 & 35 & 39 & 82 & 3 & \multirow{2}{*}{3.29} & \multirow[b]{2}{*}{0.889} & \multirow[b]{2}{*}{6} \\
\hline & & Percent & 4.3 & 32.1 & 21.0 & 40.1 & 2.5 & & & \\
\hline
\end{tabular}

Table 3. Quality Parameters for Resources - Faculty

\begin{tabular}{|c|c|c|c|c|c|c|c|c|c|c|}
\hline $\begin{array}{c}\text { S. } \\
\text { nos. }\end{array}$ & Description & & $\begin{array}{l}\text { Strongly } \\
\text { Disagree }\end{array}$ & Disagree & $\begin{array}{c}\text { No } \\
\text { opinion } \\
\end{array}$ & Agree & $\begin{array}{c}\text { Strongly } \\
\text { Agree }\end{array}$ & Mean & $\begin{array}{c}\text { Std. } \\
\text { Deviation }\end{array}$ & Rank \\
\hline \multirow[b]{2}{*}{1} & \multirow{2}{*}{$\begin{array}{c}\text { Academic and } \\
\text { professional } \\
\text { qualifications }\end{array}$} & Frequency & 0 & 2 & 43 & 37 & 80 & \multirow[b]{2}{*}{4.20} & \multirow[b]{2}{*}{0.879} & \multirow[b]{2}{*}{1} \\
\hline & & Percent & 0 & 1.2 & 26.5 & 22.8 & 49.4 & & & \\
\hline \multirow{2}{*}{2} & \multirow{2}{*}{$\begin{array}{l}\text { Teaching } \\
\text { ability and } \\
\text { experience }\end{array}$} & Frequency & 0 & 2 & 34 & 58 & 68 & \multirow{2}{*}{4.17} & \multirow{2}{*}{0.846} & \multirow{2}{*}{2} \\
\hline & & Percent & 0 & 1.2 & 21.0 & 35.8 & 42.0 & & & \\
\hline \multirow[b]{2}{*}{3} & \multirow{2}{*}{$\begin{array}{l}\text { Supervision of } \\
\text { student's } \\
\text { research }\end{array}$} & Frequency & 0 & 2 & 36 & 85 & 39 & \multirow[b]{2}{*}{3.99} & \multirow[b]{2}{*}{0.718} & \multirow[b]{2}{*}{6} \\
\hline & & Percent & & 1.2 & 22.2 & 52.5 & 24.1 & & & \\
\hline \multirow{2}{*}{4} & \multirow{2}{*}{$\begin{array}{c}\text { Innovations and } \\
\text { patents }\end{array}$} & Frequency & 0 & 9 & 45 & 90 & 18 & \multirow{2}{*}{3.72} & \multirow{2}{*}{0.733} & \multirow{2}{*}{9} \\
\hline & & Percent & & 5.6 & 27.8 & 55.6 & 11.1 & & & \\
\hline \multirow[b]{2}{*}{5} & \multirow{2}{*}{$\begin{array}{c}\text { Consultancy } \\
\text { Projects } \\
\text { undertaken }\end{array}$} & Frequency & 2 & 9 & 78 & 53 & 20 & \multirow[b]{2}{*}{3.49} & \multirow[b]{2}{*}{0.828} & \multirow[b]{2}{*}{11} \\
\hline & & Percent & 1.2 & 5.8 & 48.1 & 32.7 & 12.3 & & & \\
\hline \multirow{2}{*}{6} & \multirow{2}{*}{$\begin{array}{c}\text { Quality of } \\
\text { publications }\end{array}$} & Frequency & 0 & 16 & 46 & 57 & 43 & \multirow{2}{*}{3.78} & \multirow{2}{*}{0.950} & \multirow{2}{*}{8} \\
\hline & & Percent & & 9.9 & 28.4 & 35.2 & 26.5 & & & \\
\hline \multirow{2}{*}{7} & Faculty- & Frequency & 0 & 2 & 17 & 96 & 47 & 416 & 0640 & 3 \\
\hline & Student ratio & Percent & & 1.2 & 10.5 & 59.3 & 29.0 & 4.16 & 0.649 & 3 \\
\hline & Placement \& & Frequency & 2 & 21 & 17 & 56 & 66 & & & \\
\hline 8 & $\begin{array}{c}\text { Facilitating Job } \\
\text { Opportunities }\end{array}$ & Percent & 1.2 & 13.0 & 10.5 & 34.6 & 40.7 & 4.01 & 1.072 & 5 \\
\hline 0 & Professional & Frequency & 2 & 12 & 23 & 75 & 50 & & 0900 & \\
\hline 9 & Practices & Percent & 1.2 & 7.4 & 14.2 & 46.3 & 30.9 & 3.98 & 0.929 & 7 \\
\hline & Participation in & Frequency & 0 & 17 & 54 & 67 & 24 & & & \\
\hline 10 & $\begin{array}{c}\text { the } \\
\text { Organizational } \\
\text { Activities }\end{array}$ & Percent & 0 & 10.5 & 33.3 & 41.4 & 14.8 & 3.60 & 0.866 & 10 \\
\hline & Membership of & Frequency & 2 & 26 & 58 & 65 & 11 & & & \\
\hline 11 & $\begin{array}{c}\text { professional } \\
\text { bodies }\end{array}$ & Percent & 1.2 & 16.0 & 35.8 & 40.1 & 6.8 & 3.35 & 0.874 & 12 \\
\hline & Social & Frequency & 0 & 6 & 41 & 55 & 60 & & & \\
\hline 12 & Responsibility & Percent & 0 & 3.7 & 25.3 & 34.0 & 37.0 & 4.04 & 0.880 & 4 \\
\hline
\end{tabular}


Table 4. Quality Parameters for Resources - Infrastructure

\begin{tabular}{|c|c|c|c|c|c|c|c|c|c|c|}
\hline $\begin{array}{c}\text { S. } \\
\text { nos. }\end{array}$ & Description & & $\begin{array}{l}\text { Strongly } \\
\text { Disagree }\end{array}$ & Disagree & $\begin{array}{c}\text { No } \\
\text { opinion }\end{array}$ & Agree & $\begin{array}{c}\text { Strongly } \\
\text { Agree }\end{array}$ & Mean & $\begin{array}{c}\text { Std. } \\
\text { Deviation }\end{array}$ & Rank \\
\hline \multirow[b]{2}{*}{1} & \multirow{2}{*}{$\begin{array}{l}\text { Classrooms, } \\
\text { offices, } \\
\text { laboratories, } \\
\text { and associated } \\
\text { equipment }\end{array}$} & Frequency & 0 & 0 & 7 & 36 & 119 & \multirow[b]{2}{*}{4.69} & \multirow[b]{2}{*}{$0 . .549$} & \multirow[b]{2}{*}{1} \\
\hline & & Percent & 0 & 0 & 4.3 & 22.2 & 73.5 & & & \\
\hline \multirow{2}{*}{2} & \multirow{2}{*}{$\begin{array}{l}\text { Modern tools, } \\
\text { equipment, } \\
\text { computing } \\
\text { resources }\end{array}$} & Frequency & 0 & 9 & 3 & 51 & 99 & \multirow{2}{*}{4.48} & \multirow{2}{*}{0.790} & \multirow{2}{*}{4} \\
\hline & & Percent & 0 & 5.6 & 1.9 & 31.5 & 61.1 & & & \\
\hline \multirow{2}{*}{3} & \multirow{2}{*}{$\begin{array}{c}\text { library } \\
\text { Services } \\
\text { \&amp; } \\
\text { E-Resources }\end{array}$} & Frequency & 2 & 0 & 7 & 55 & 98 & \multirow{2}{*}{4.52} & \multirow{2}{*}{0.698} & \multirow{2}{*}{2} \\
\hline & & Percent & 0 & 5.6 & 1.9 & 31.5 & 61.1 & & & \\
\hline \multirow{2}{*}{4} & \multirow{2}{*}{$\begin{array}{c}\text { Technical } \\
\text { Support }\end{array}$} & Frequency & 0 & 0 & 10 & 63 & 89 & \multirow{2}{*}{4.49} & \multirow{2}{*}{0.613} & \multirow{2}{*}{3} \\
\hline & & Percent & 0 & & 6.2 & 38.9 & 54.9 & & & \\
\hline
\end{tabular}

Table 5. Quality Parameters for Education Management

\begin{tabular}{|c|c|c|c|c|c|c|c|c|c|c|}
\hline $\begin{array}{c}\text { S. } \\
\text { nos. }\end{array}$ & Description & & $\begin{array}{l}\text { Strongly } \\
\text { Disagree }\end{array}$ & Disagree & $\begin{array}{c}\text { No } \\
\text { opinion }\end{array}$ & Agree & $\begin{array}{c}\text { Strongly } \\
\text { Agree }\end{array}$ & Mean & $\begin{array}{c}\text { Std. } \\
\text { Deviation } \\
\end{array}$ & Rank \\
\hline \multirow{2}{*}{1} & \multirow{2}{*}{$\begin{array}{c}\text { Institutional } \\
\text { Vision \& } \\
\text { Mission }\end{array}$} & Frequency & 0 & 0 & 3 & 74 & 85 & \multirow{2}{*}{4.51} & \multirow{2}{*}{0.537} & \multirow{2}{*}{3} \\
\hline & & Percent & 0 & 0 & 1.9 & 45.7 & 52.5 & & & \\
\hline \multirow[b]{2}{*}{2} & \multirow{2}{*}{$\begin{array}{l}\text { Management } \\
\text { Support for } \\
\text { Infrastructure } \\
\text { Facilitation }\end{array}$} & Frequency & 0 & 0 & 0 & 77 & 85 & \multirow[b]{2}{*}{4.52} & \multirow[b]{2}{*}{0.501} & \multirow[b]{2}{*}{2} \\
\hline & & Percent & 0 & 0 & & 47.5 & 52.5 & & & \\
\hline \multirow{2}{*}{3} & \multirow{2}{*}{$\begin{array}{l}\text { Finance and } \\
\text { Management }\end{array}$} & Frequency & 0 & 0 & 17 & 75 & 70 & \multirow{2}{*}{4.33} & \multirow{2}{*}{0.658} & \multirow{2}{*}{6} \\
\hline & & Percent & 0 & 0 & 10.5 & 46.3 & 43.2 & & & \\
\hline \multirow[t]{2}{*}{4} & \multirow{2}{*}{$\begin{array}{l}\text { Governance } \\
\text { \&amp; } \\
\text { Institutional } \\
\text { support } \\
\end{array}$} & Frequency & 10 & 3 & 10 & 80 & 59 & \multirow[t]{2}{*}{4.08} & \multirow[t]{2}{*}{1.027} & \multirow[t]{2}{*}{7} \\
\hline & & Percent & 6.2 & 1.9 & 6.2 & 49.4 & 36.4 & & & \\
\hline \multirow{2}{*}{5} & \multirow{2}{*}{$\begin{array}{c}\text { Education } \\
\text { Environment }\end{array}$} & Frequency & 0 & 0 & 7 & 62 & 93 & \multirow{2}{*}{4.53} & \multirow{2}{*}{0.581} & \multirow{2}{*}{1} \\
\hline & & Percent & 0 & 0 & 4.3 & 38.3 & 57.4 & & & \\
\hline \multirow{2}{*}{6} & \multirow{2}{*}{$\begin{array}{l}\text { Continuous } \\
\text { improvement }\end{array}$} & Frequency & 0 & 0 & 11 & 62 & 89 & \multirow{2}{*}{4.48} & \multirow{2}{*}{0.623} & \multirow{2}{*}{5} \\
\hline & & Percent & 0 & 0 & 6.8 & 38.3 & 54.9 & & & \\
\hline \multirow{2}{*}{7} & \multirow{2}{*}{$\begin{array}{c}\text { Quality } \\
\text { Management }\end{array}$} & Frequency & 0 & 0 & 0 & 83 & 79 & \multirow{2}{*}{4.49} & \multirow{2}{*}{0.501} & \multirow{2}{*}{4} \\
\hline & & Percent & 0 & 0 & 0 & 51.2 & 48.8 & & & \\
\hline
\end{tabular}


Table 6. Quality Parameters for Curriculum Content \& Quality

\begin{tabular}{|c|c|c|c|c|c|c|c|c|c|c|}
\hline $\begin{array}{l}\text { S. } \\
\text { nos. }\end{array}$ & Description & & $\begin{array}{l}\text { Strongly } \\
\text { Disagree }\end{array}$ & Disagree & $\begin{array}{c}\text { No } \\
\text { opinion }\end{array}$ & Agree & $\begin{array}{c}\text { Strongly } \\
\text { Agree }\end{array}$ & Mean & $\begin{array}{c}\text { Std. } \\
\text { Deviation }\end{array}$ & Rank \\
\hline \multirow[b]{2}{*}{1} & \multirow{2}{*}{$\begin{array}{l}\text { Approach \& } \\
\text { Methodology for } \\
\text { Qualifying } \\
\text { Curriculum } \\
\text { Content }\end{array}$} & Frequency & 0 & 0 & 0 & 89 & 73 & \multirow[b]{2}{*}{4.45} & \multirow[b]{2}{*}{0.499} & \multirow[b]{2}{*}{1} \\
\hline & & Percent & 0 & 0 & 0 & 54.9 & 45.1 & & & \\
\hline \multirow{2}{*}{2} & \multirow{2}{*}{$\begin{array}{l}\text { Programme } \\
\text { Structure and } \\
\text { Content }\end{array}$} & Frequency & 0 & 0 & 7 & 92 & 63 & \multirow{2}{*}{4.35} & \multirow{2}{*}{0.561} & \multirow{2}{*}{3} \\
\hline & & Percent & 0 & 0 & 4.3 & 56.8 & 38.9 & & & \\
\hline \multirow{2}{*}{3} & \multirow{2}{*}{$\begin{array}{l}\text { Student-centred } \\
\text { learning } \\
\text { environment } \\
\end{array}$} & Frequency & 0 & 0 & 32 & 58 & 72 & \multirow{2}{*}{4.25} & \multirow{2}{*}{0.765} & \multirow{2}{*}{4} \\
\hline & & Percent & 0 & 0 & 19.8 & 35.8 & 44.4 & & & \\
\hline \multirow{2}{*}{4} & \multirow{2}{*}{$\begin{array}{l}\text { Flexible } \\
\text { curriculum }\end{array}$} & Frequency & 0 & 3 & 52 & 66 & 41 & \multirow{2}{*}{3.90} & \multirow{2}{*}{0.801} & \multirow{2}{*}{8} \\
\hline & & Percent & 0 & 1.9 & 32.1 & 40.7 & 25.3 & & & \\
\hline \multirow{2}{*}{5} & \multirow{2}{*}{$\begin{array}{l}\text { Disciplines and } \\
\text { cross-disciplinary } \\
\text { modules }\end{array}$} & Frequency & 10 & 19 & 24 & 52 & 57 & \multirow{2}{*}{3.78} & \multirow{2}{*}{1.220} & \multirow{2}{*}{9} \\
\hline & & Percent & 6.2 & 11.7 & 14.8 & 32.1 & 35.2 & & & \\
\hline \multirow{2}{*}{6} & \multirow{2}{*}{$\begin{array}{l}\text { Complementary } \\
\text { Studies }\end{array}$} & Frequency & 10 & 29 & 57 & 43 & 23 & \multirow{2}{*}{3.25} & \multirow{2}{*}{1.098} & \multirow{2}{*}{10} \\
\hline & & Percent & 6.2 & 17.9 & 35.2 & 26.5 & 14.2 & & & \\
\hline \multirow{2}{*}{7} & \multirow{2}{*}{$\begin{array}{l}\text { Educational } \\
\text { Methods }\end{array}$} & Frequency & 0 & 6 & 23 & 74 & 59 & \multirow{2}{*}{4.15} & & \\
\hline & & Percent & 0 & 3.7 & 14.2 & 45.7 & 36.4 & & 0.170 & 0 \\
\hline 8 & & Frequency & 0 & & 20 & 82 & 59 & 425 & 0660 & \\
\hline 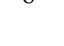 & 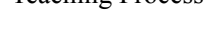 & Percent & 0 & & 12.3 & 50.6 & 37 & 1.20 & 0.000 & 1 \\
\hline & & Frequency & 0 & & 20 & 12.3 & 12.3 & & & \\
\hline & 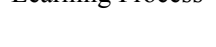 & Percent & 0 & 0 & 12.3 & 34.0 & 53.7 & 4.41 & 0.102 & 2 \\
\hline 10 & Doreg h n. & Frequency & 0 & 9 & 20 & 75 & 58 & 112 & $002 ?$ & \\
\hline & 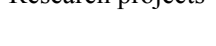 & Percent & 0 & 5.6 & 12.3 & 46.3 & 35.8 & 1.12 & 0.052 & $r$ \\
\hline
\end{tabular}

Table 7. Quality Parameters for Assessment \& Evaluation

\begin{tabular}{|c|c|c|c|c|c|c|c|c|c|c|}
\hline $\begin{array}{c}\text { S. } \\
\text { nos. }\end{array}$ & Description & & $\begin{array}{l}\text { Strongly } \\
\text { Disagree }\end{array}$ & Disagree & $\begin{array}{c}\text { No } \\
\text { opinion }\end{array}$ & Agree & $\begin{array}{c}\text { Strongly } \\
\text { Agree }\end{array}$ & Mean & $\begin{array}{c}\text { Std. } \\
\text { Deviation }\end{array}$ & Rank \\
\hline \multirow{2}{*}{1} & \multirow{2}{*}{$\begin{array}{l}\text { Students } \\
\text { Performance } \\
\text { Evaluation }\end{array}$} & Frequency & 0 & 0 & 28 & 67 & 67 & \multirow{2}{*}{4.24} & \multirow{2}{*}{0.729} & \multirow{2}{*}{2} \\
\hline & & Percent & 0 & 0 & 17.3 & 41.4 & 41.4 & & & \\
\hline \multirow{2}{*}{2} & \multirow{2}{*}{$\begin{array}{c}\text { Examination } \\
\text { System }\end{array}$} & Frequency & 0 & 31 & 23 & 64 & 44 & \multirow{2}{*}{3.75} & \multirow{2}{*}{1.059} & \multirow{2}{*}{5} \\
\hline & & Percent & 0 & 19.1 & 14.2 & 39.5 & 27.2 & & & \\
\hline \multirow{2}{*}{3} & \multirow{2}{*}{$\begin{array}{l}\text { Assessment } \\
\text { Strategies }\end{array}$} & Frequency & 0 & 10 & 9 & 90 & 53 & \multirow{2}{*}{4.15} & \multirow{2}{*}{0.782} & \multirow{2}{*}{3} \\
\hline & & Percent & 0 & 6.2 & 5.6 & 55.6 & 32.7 & & & \\
\hline \multirow[t]{2}{*}{4} & \multirow[t]{2}{*}{$\begin{array}{l}\text { Development } \\
\text { plan for Under } \\
\text { performers }\end{array}$} & Frequency & 0 & 3 & 6 & 100 & 53 & \multirow[t]{2}{*}{4.25} & \multirow[t]{2}{*}{0.614} & \multirow[t]{2}{*}{1} \\
\hline & & Percent & & 1.9 & 3.7 & 61.7 & 32.7 & & & \\
\hline \multirow{2}{*}{5} & \multirow{2}{*}{$\begin{array}{l}\text { Achievement } \\
\text { of Learning } \\
\text { Outcomes }\end{array}$} & Frequency & 0 & 9 & 21 & 73 & 59 & \multirow{2}{*}{4.12} & \multirow{2}{*}{0.840} & \multirow{2}{*}{4} \\
\hline & & Percent & 0 & 5.6 & 13.0 & 45.1 & 36.4 & & & \\
\hline
\end{tabular}


Table 8. Quality Parameters for Learning Outcomes

\begin{tabular}{|c|c|c|c|c|c|c|c|c|c|c|}
\hline $\begin{array}{c}\text { S. } \\
\text { nos. }\end{array}$ & Description & & $\begin{array}{l}\text { Strongly } \\
\text { Disagree }\end{array}$ & Disagree & $\begin{array}{c}\text { No } \\
\text { opinion }\end{array}$ & Agree & $\begin{array}{c}\text { Strongly } \\
\text { Agree }\end{array}$ & Mean & $\begin{array}{c}\text { Std. } \\
\text { Deviation }\end{array}$ & Rank \\
\hline \multirow{2}{*}{1} & \multirow{2}{*}{$\begin{array}{l}\text { Ability to apply } \\
\text { knowledge }\end{array}$} & Frequency & 0 & 0 & 14 & 35 & 113 & \multirow{2}{*}{4.61} & \multirow{2}{*}{0.499} & \multirow{2}{*}{1} \\
\hline & & Percent & 0 & 0 & 8.6 & 21.6 & 69.8 & & & \\
\hline \multirow[b]{2}{*}{2} & \multirow{2}{*}{$\begin{array}{l}\text { Ability to } \\
\text { design and } \\
\text { conduct } \\
\text { experiments }\end{array}$} & Frequency & 0 & 0 & 21 & 83 & 58 & \multirow[b]{2}{*}{4.23} & \multirow[b]{2}{*}{0.561} & \multirow[b]{2}{*}{2} \\
\hline & & Percent & 0 & 0 & 13.0 & 51.2 & 35.8 & & & \\
\hline \multirow{2}{*}{3} & \multirow{2}{*}{$\begin{array}{c}\text { Ability to } \\
\text { design a system }\end{array}$} & Frequency & 0 & 7 & 38 & 62 & 55 & \multirow{2}{*}{4.02} & \multirow{2}{*}{0.765} & \multirow{2}{*}{6} \\
\hline & & Percent & 0 & 4.3 & 23.5 & 38.3 & 34.0 & & & \\
\hline \multirow{2}{*}{4} & \multirow{2}{*}{$\begin{array}{l}\text { Knowledge of } \\
\text { contemporary } \\
\text { issues }\end{array}$} & Frequency & 0 & 6 & 22 & 82 & 52 & \multirow{2}{*}{4.11} & \multirow{2}{*}{0.801} & \multirow{2}{*}{4} \\
\hline & & Percent & 0 & 3.7 & 13.6 & 50.6 & 32.1 & & & \\
\hline \multirow{2}{*}{5} & \multirow{2}{*}{$\begin{array}{l}\text { Professional } \\
\text { qualifications }\end{array}$} & Frequency & 0 & 6 & 38 & 82 & 36 & \multirow{2}{*}{3.91} & \multirow{2}{*}{1.220} & \multirow{2}{*}{7} \\
\hline & & Percent & & 3.7 & 23.5 & 50.6 & 22.2 & & & \\
\hline \multirow{2}{*}{6} & \multirow{2}{*}{$\begin{array}{l}\text { Results of } \\
\text { outcome } \\
\text { assessment }\end{array}$} & Frequency & 2 & 6 & 43 & 74 & 37 & \multirow{2}{*}{3.85} & \multirow{2}{*}{1.098} & \multirow{2}{*}{8} \\
\hline & & Percent & 1.2 & 3.7 & 26.5 & 45.7 & 22.8 & & & \\
\hline \multirow{2}{*}{7} & \multirow{2}{*}{$\begin{array}{l}\text { Programme } \\
\text { Outcomes }\end{array}$} & Frequency & 2 & 6 & 36 & 50 & 68 & \multirow{2}{*}{4.09} & \multirow{2}{*}{0.798} & \multirow{2}{*}{5} \\
\hline & & Percent & 1.2 & 3.7 & 22.2 & 30.9 & 42.0 & & & \\
\hline & Discipline & Frequency & 0 & 6 & 25 & 69 & 62 & & & \\
\hline 0 & based criteria & Percent & 0 & 3.7 & 15.4 & 42.6 & 38.3 & 4.15 & 0.000 & 3 \\
\hline
\end{tabular}

Table 9. Quality Parameters for Generic skills and Learning Experiences

\begin{tabular}{|c|c|c|c|c|c|c|c|c|c|c|}
\hline $\begin{array}{c}\text { S. } \\
\text { nos. }\end{array}$ & Description & & $\begin{array}{l}\text { Strongly } \\
\text { Disagree }\end{array}$ & Disagree & $\begin{array}{c}\text { No } \\
\text { opinion } \\
\end{array}$ & Agree & $\begin{array}{c}\text { Strongly } \\
\text { Agree }\end{array}$ & Mean & $\begin{array}{c}\text { Std. } \\
\text { Deviation }\end{array}$ & Rank \\
\hline \multirow{2}{*}{1} & \multirow{2}{*}{$\begin{array}{l}\text { Developing } \\
\text { Problem } \\
\text { solving skills }\end{array}$} & Frequency & 0 & 0 & 44 & 55 & 63 & \multirow{2}{*}{4.12} & \multirow{2}{*}{0.807} & \multirow{2}{*}{5} \\
\hline & & Percent & 0 & 0 & 27.2 & 34.0 & 38.9 & & & \\
\hline \multirow{2}{*}{2} & \multirow{2}{*}{$\begin{array}{c}\text { Developing } \\
\text { ability to } \\
\text { work as team } \\
\text { member }\end{array}$} & Frequency & 0 & 0 & 19 & 55 & 88 & \multirow{2}{*}{4.43} & \multirow{2}{*}{0.694} & \multirow{2}{*}{1} \\
\hline & & Percent & 0 & 0 & 11.7 & 34.0 & 54.3 & & & \\
\hline \multirow{2}{*}{3} & \multirow{2}{*}{$\begin{array}{l}\text { Developing } \\
\text { ability to plan }\end{array}$} & Frequency & 0 & 0 & 31 & 65 & 66 & \multirow{2}{*}{4.22} & \multirow{2}{*}{0.745} & \multirow{2}{*}{4} \\
\hline & & Percent & 0 & 0 & 19.1 & 40.1 & 40.7 & & & \\
\hline \multirow{2}{*}{4} & \multirow{2}{*}{$\begin{array}{c}\text { Confidence to } \\
\text { learning }\end{array}$} & Frequency & 0 & 6 & 22 & 63 & 71 & \multirow{2}{*}{4.23} & \multirow{2}{*}{0.821} & \multirow{2}{*}{3} \\
\hline & & Percent & 0 & 3.7 & 13.6 & 38.9 & 43.8 & & & \\
\hline \multirow{2}{*}{5} & \multirow{2}{*}{$\begin{array}{l}\text { Achieving the } \\
\text { goals }\end{array}$} & Frequency & 0 & 8 & 45 & 75 & 34 & \multirow{2}{*}{3.83} & \multirow{2}{*}{0.813} & \multirow{2}{*}{6} \\
\hline & & Percent & 0 & 4.9 & 27.8 & 46.3 & 21.0 & & & \\
\hline \multirow{2}{*}{6} & \multirow{2}{*}{$\begin{array}{c}\text { Exploring } \\
\text { new } \\
\text { opportunities } \\
\text { in life }\end{array}$} & Frequency & 0 & 0 & 25 & 55 & 82 & \multirow{2}{*}{4.35} & \multirow{2}{*}{0.735} & \multirow{2}{*}{2} \\
\hline & & Percent & 0 & 0 & 15.4 & 34.0 & 50.6 & & & \\
\hline
\end{tabular}


Table 10. Skillset Requirement for Students

\begin{tabular}{|c|c|c|c|c|c|c|c|c|c|c|}
\hline $\begin{array}{c}\text { S. } \\
\text { nos. }\end{array}$ & Description & & $\begin{array}{l}\text { Strongly } \\
\text { Disagree }\end{array}$ & Disagree & $\begin{array}{c}\begin{array}{c}\text { No } \\
\text { opinion }\end{array} \\
\end{array}$ & Agree & $\begin{array}{c}\begin{array}{c}\text { Strongly } \\
\text { Agree }\end{array} \\
\end{array}$ & Mean & $\begin{array}{c}\text { Std. } \\
\text { Deviation }\end{array}$ & Rank \\
\hline \multirow{2}{*}{1} & \multirow{2}{*}{ Analytical skills } & Frequency & 0 & 1 & 44 & 59 & 58 & \multirow{2}{*}{4.17} & \multirow{2}{*}{0.710} & \multirow{2}{*}{4} \\
\hline & & Percent & 0 & 6 & 27.2 & 36.4 & 35.8 & & & \\
\hline \multirow{2}{*}{2} & \multirow{2}{*}{ Creativity } & Frequency & 0 & 0 & 18 & 28 & 116 & \multirow{2}{*}{4.40} & \multirow{2}{*}{0.635} & \multirow{2}{*}{1} \\
\hline & & Percent & 0 & 0 & 11.1 & 17.3 & 71.6 & & & \\
\hline \multirow{2}{*}{3} & \multirow{2}{*}{ Lateral thinking } & Frequency & 0 & 6 & 22 & 62 & 72 & \multirow{2}{*}{4.16} & \multirow{2}{*}{0.787} & \multirow{2}{*}{5} \\
\hline & & Percent & 0 & 3.7 & 13.6 & 38.3 & 44.4 & & & \\
\hline \multirow{2}{*}{4} & \multirow{2}{*}{ Problem solving } & Frequency & 0 & 6 & 36 & 64 & 56 & \multirow{2}{*}{4.31} & \multirow{2}{*}{0.733} & \multirow{2}{*}{2} \\
\hline & & Percent & 0 & 3.7 & 22.2 & 39.5 & 34.6 & & & \\
\hline \multirow{2}{*}{5} & \multirow{2}{*}{ Communication } & Frequency & 0 & 6 & 29 & 63 & 65 & \multirow{2}{*}{4.22} & \multirow{2}{*}{0.720} & \multirow{2}{*}{3} \\
\hline & & Percent & 0 & 3.1 & 17.9 & 38.9 & 40.1 & & & \\
\hline \multirow{2}{*}{6} & \multirow{2}{*}{$\begin{array}{l}\text { Aesthetic } \\
\text { sensibility }\end{array}$} & Frequency & 0 & 9 & 56 & 48 & 49 & \multirow{2}{*}{3.59} & \multirow{2}{*}{1.025} & \multirow{2}{*}{8} \\
\hline & & Percent & 0 & 5.6 & 34.6 & 29.6 & 30.2 & & & \\
\hline \multirow{2}{*}{7} & \multirow{2}{*}{ Extend learning } & Frequency & 0 & 5 & 75 & 58 & 24 & \multirow{2}{*}{3.65} & \multirow{2}{*}{0.908} & \multirow{2}{*}{7} \\
\hline & & Percent & 0 & 3.1 & 46.3 & 35.9 & 14.8 & & & \\
\hline & Digital and & Frequency & 0 & 8 & 10 & 108 & 36 & & & \\
\hline 0 & knowledge & Percent & 0 & 4.9 & 6.2 & 66.7 & 22.2 & 4.09 & 0.034 & 0 \\
\hline
\end{tabular}

The sample comprises $67.3 \%$ of males and $32.7 \%$ of female. Among the 162 sample participants, $48.2 \%$ were assistant professors, $33.3 \%$ associate professors and $18.5 \%$ professors.

The tables 2 to 10 show the quality parameters and its ranking preferences. The vital factors that hold significance in maintaining the quality assurance in fashion technology institutes are students' admission policies and regulations, mentoring which means resources for academic advice for students along with the academic and professional qualifications of the faculty. Moreover, the infrastructure facilities like classrooms, offices, laboratories, and equipment have been considered essential parameters in combination with the management support for infrastructure facilitation. The approach with the methodology for the qualifying curriculum content has significance in sustaining the quality with student's ability for application of knowledge. The other attributes that were considered vital are professional practical approach as well as creativity. The parameters with the least significance identified were the admission of students from other countries, membership of professional bodies, modern tools and computing resources, governance and institutional support, complementary studies, results of outcome assessment, achieving the goals, and Aesthetic sensibility examination system, entrepreneurship, and extended learning.

Based on the data analysis, various test results using SPSS were attained which represented the responses for the study objectives identified. The findings from the performed tests portray that there has been a significant influence of the provided amenities to students as well as the faculty. Moreover, there has been a vital effect of the infrastructure and the designed curriculum and are considered important parameters in quality assurance. The professional attribute is also a significant contributor to maintaining the quality parameters within the institute. The skillset is also recognized as a crucial factor for sustaining quality aspects with the institutional framework.

\subsection{Inferences}

The various aspects that were inferred from the analysis have assisted in comparing with the literature reviewed. As per prior studies referred, the various agencies for maintaining quality assurance in higher education are found significant. From the analysis results as well, the curriculum designed as per the standards mentioned by these agencies plays a contributing role in maintaining the quality level in government institutes. The infrastructure facilities and amenities provided to the students and faculties emerged as vital quality parameters as per the analysis and were also identified significantly in the framework studied in the prior literature studies. The quality assurance models highlighted through the literature review mentioned the crucial components like professional attributes, decision making, students and faculty involvement, management support for the success of the quality assurance in higher education and similar parameters were identified through the data analysis as per the data collected from the respondents. The research findings have motivated in developing distinct strategies that can be employed or strengthened if already existing, 
in enhancing and maintaining the quality assurance levels for the progress of the fashion technology institutes to boost the existence of quality institutes within the segment of higher education. It has also emphasized the importance of the challenges and the corresponding strategies to conquer them which facilitated the suitable strategies to be used appropriately with support in marking their presence. Hence the results discussed in this section depict that the research objectives and the questions were accomplished.

\section{Conclusion and Recommendation}

In India, the Higher Education segment has observed an incredible boost in the amount of Universities \& Colleges and students' enrolment but the quality is still an area of apprehension till now, which has been an indispensable component in achieving the aim and execution of national policy. At once, there has been a higher demand for accessing higher education, and at the same time, the quality parameters are under question. Managing quality in higher educational institutes has never been the same as that of business and industry. Higher educational institutes act as the society that considers the decision of maintaining standards and quality. The educational process is also developed based on society collaborative learning, where the students are required to participate with an active responsibility. For surviving in the global world of competition, all higher education institutions are required to focus especially on quality assurance in higher education.

This study is also intended to identify the quality assurance parameters with further development of strategies as well as a framework based on the determined parameters in fashion technology institutes in the context of higher education. The prior works of literature available in this domain were discussed and the outcomes were analysed with the obtained results from the analysis of the collected data, done as part of the study. To gain insights into the conceptual advancements and important quality assurance parameters, different characteristics of quality were examined and diverse techniques of quality assurance that comprises quality frameworks, best practices, and external quality monitoring agencies were discussed. The external quality monitoring agencies have been recognized as the general way of quality assurance, though a quality framework is considered to be in-built in the process. The diverse models of quality assurance are accessible and were reviewed. Moreover, the existing issues along with the challenges associated with the maintenance of quality assurance practices were highlighted. The various parameters revealed as part of the data collection and analysis can help in maintaining the quality with the fashion technological institutes with assisting in developing strategies to improve the quality of higher education in India. The significant parameters for quality higher education are quality faculty members, student participation, the infrastructure of the educational institutions, curriculum, learning resources, management support, and institutional leadership.

This research has supported innovative opportunities and trends to further research in this area by mapping the research gap recognized while reviewing the existing literature in the literature review section. The complete study mainly focuses on the quality assurance practices of higher educational institutes referred by the previous researchers and provides the prospects for the quality assurance review practices that can be followed for enhancement in the quality levels in the domain of fashion technology and higher education. This study was eventually successful in meeting the results acquired from the participants with the research questions and objectives. The practical inferences of this study are discussed with inclusions of recommendations to facilitate the expansion of the scope for future research.

\section{REFERENCES}

[1] Al-Atiqi, I. M., \& Deshpande, P. B. Transforming higher education with six sigma. In International network of Quality Assessment Agencies in Higher Education, Biannual Conference in Abu Dhabi (Vol. 30), 2009. https://www.researchgate.net/publication/236201448_Quali ty_in_Higher_Education_United_Arab_Emirates_Perspecti ve

[2] Alena D. Matraeva, Marina V. Rybakova1, Mikhail V. Vinichenko, Natalia V. Ljapunova, Aleksander A. Oseev. Development of Creativity of Students in Higher Educational Institutions: Assessment of Students and Experts, Universal Journal of Educational Research Vol. 8(1), 2020.https://www.hrpub.org/journals/article_info.php ?aid $=8661$

[3] J. Bennedsen, S. Rouvrais, J. Roslöf, J. Kontio, F. Georgsson \& C. D. McCartan. Collaborative quality enhancement in engineering education: an overview of operational models at a programme level, (Vol. 45), 2020. https://www.tandfonlin e.com/doi/full/10.1080/03043797.2018.1443058

[4] Ashvin Dhawan,. Let's start at the very beginning https://www.stanforddaily.com/2018/08/02/lets-start-at-thevery-beginning/

[5] Bordia, S. Problems of accreditation and quality assurance of engineering education in developing countries. European journal of engineering education, 26(2), 187-193, 2001. https://www.tandfonline.com/doi/abs/10.1080/0304379011 0034447 ,

[6] Creswell, J. W., \& Creswell, J. D. Research design: Qualitative, quantitative, and mixed methods approaches. Sage publications. 2017. file://D:/user\%20data/Downloads/ 8530-Article\%20Text-20365-2-10-20120307\%20(2).pdf

[7] Dhanuraj, D., \& Kumar, R. V. (2015). Understanding the 
Status of Higher Education in India: Challenges and Scepticism towards Serious Investments in the Sector. Centre for Public Policy Research. https://www.cppr.in/wpcontent/uploads/2015/01/Higher-Education.pdf

[8] Dill, D. Quality assurance in higher education: Practices and issues. in-Chief Barry McGaw, Eva Baker and Penelope P. Peterson, Elsevier Publications. 2007.https://www.scienced irect.com/referencework/9780080448947/international-enc yclopedia-of-education

[9] Dr.Mohan Lal. "Quality Parameters of National Assessment and Accreditation Council (NAAC) \& Teacher Education", Dr. B. R. Ambedkar College of Education, Opp. New Bus Stand (Kheri Markanda) Kurukshetra, Haryana-136118. 2017.

http://www.naac.gov.in/docs/Books/Quality\%20Enhancem ent $\% 20$ in\%20Teacher\%20Education.pdf

[10] Duarte, D., \& Martins, P. V. A maturity model for higher education institutions. Journal of Spatial and Organizational Dynamics, 1(1), 25-44. 2013. https://ideas.repec.org/a/ris/js pord/0002.html

[11] Gnanam, A., \& Chairman, N. E. Globalization and its impact on quality assurance, accreditation and recognition of qualifications. Accessed on, 29. 2008.https://www.research gate.net/publication/272474607__QQuality_assurance_in_hi gher_education_Comparative analysis_of_provisions_and practices_in_Ghana_and_New_Zealand

[12] Honnutagi, A. R., Sonar, R., \& Babu, A. S. Achieving quality excellence in Indian engineering education: modelling and analysis using system dynamics. International Journal of Business Excellence, 10(1), 90-119. 2016. https://core.ac.uk/download/pdf/55305275.pdf

[13] Kahveci, T. C., Uygun, Ö., Yurtsever, U., \& İlyas, S. Quality assurance in higher education institutions using strategic information systems. Procedia-Social and behavioral sciences, 55, 161-167. 2012. https://www.scienc edirect.com/science/article/pii/S1877042812039523

[14] Khan, Z., Role of National Assessment and Accreditation Council (NAAC) and Its Benefits, 2019. http://www.yourar ticlelibrary.com/education/role-of-national-assessment-andaccreditation-council-naac-and-its-benefits/45185

[15] Mehta, N., Verma, P., \& Seth, N. Total quality management implementation in engineering education in India: an interpretive structural modelling approach. Total Quality Management \& Business Excellence, 25(1-2), 124-140. 2014.

https://www.tandfonline.com/doi/abs/10.1080/14783363.20 13.791113

[16] Mishra, S. Quality assurance in higher education: An introduction. National Assessment and Accreditation Council, India. 2007. http://oasis.col.org/handle/11599/101

[17] P N Pabrekar*, B B Sharma, M B Kekare , and S S Barve, Quality Assurance in Higher Education Institutions in India, 2nd International Conference on Law, Education and Humanities (ICLEH'15). 2015. https://icehm.org/upload/62 97ED0515562.pdf

[18] Parth Welankar, All educational institutions in India to be Naac accredited by 2022: ministry. 2019. https://www.hind ustantimes.com/pune-news/all-educational-in-india-to-be-n aac-accredited-by-2022-ministry/story-6aj5NSXHkruvN1xI
1A4tAI.html

[19] Pineda, A. P. M. Total quality management in educational institutions: Influences on customer satisfaction. Asian Journal of Management Sciences and Education, 2(3). 2013. https://pdfs.semanticscholar.org/343d/86b3e085c67788b83 8b451c9129ace77424c.pdf

[20] Press Information Bureau Government of India, Quality, Accreditation and Ranking - A Silent Revolution in the Offing in Indian Higher Education. 2019. http://pib.nic.in/newsite/PrintRelease.aspx?relid=188620

[21] Ryan, T. Quality assurance in higher education: A review of literature. 2015.https://www.hlrcjournal.com/index.php/HL $\mathrm{RC} /$ article/view/257

[22] Saeki, H., \& Imaizumi, S. International comparative study: engineering education in India. 2013. http://documents.worl dbank.org/curated/en/586761468044369699/International-c omparative-study-engineering-education-in-India

[23] Sakunth Kumar, NAAC - An Overview, Top NAAC Colleges \& Universities, Benefits, Grading System. 2018. https://www.collegedekho.com/articles/national-assessment -and-accreditation-council-naac/

[24] Senthilkumar, N., \& Arulraj, A. SQM-HEI-determination of service quality measurement of higher education in India. Journal of Modelling in Management, 6(1), 60-78. 2011. https:/www.emeraldinsight.com/doi/abs/10.1108/17465661 111112502

[25] Singh, M. Reinserting the 'public good 'into higher education transformation. Kagisano Higher Education Discussion Series, 1, 8-18. 2001. http://citeseerx.ist.psu.edu /viewdoc/download?doi=10.1.1.470.8247\&rep=rep1\&type $=\mathrm{pdf}$

[26] Stella, A., \& Bhushan, S. Quality assurance of transnational higher education: The experiences of Australia and India. Australian Universities Quality Agency and the National University of Educational Planning and Administration. 2011.

http://www.nuepa.org/new/Download/Publications/Quality $\% 20$ Assurance $\% 20$ of $\% 20$ Transnational $\% 20$ Higher $\% 20$ Edu cation.PDF

[27] Van Zanten, M., Norcini, J. J., Boulet, J. R., \& Simon, F. Overview of accreditation of undergraduate medical education programmes worldwide. Medical education, 42(9), 930-937. 2008.

[28] Venkatesh, P. Accreditation as a Benchmark of Quality Sustenance in Higher Education: A Review of Quality Assurance in Indian University System. Nitte Management Review, 7(1), 59-67. 2013. http://www.informaticsjournals. com/index.php/nmr/article/view/18414

[29] Verma, A. A review of quality assurance in higher education institutions. IMPACT Journals, International Journal of Research in Humanities, Arts and Literature (IMPACT: IJRHAL), ISSN (P), 2347-4564. 2016. http://oaji.net/articl es/2016/488-1466244455.pdf

[30] West, J. Quality management principles: Foundation of ISO 9000: 2000 family. Quality Progress, 33(2), 113. 2000. http://asq.org/quality-progress/2000/02/standards-outlook/q uality-management-principles--foundation-of-iso-9000-200 0 -family.html 
[31] Zaki, S., \& Rashidi, M. Z. Parameters of Quality in Higher Education: A Theoretical Framework. International Journal of Social Sciences \& Education, 3(4). 2013. http://ijsse.com/sites/default/files/issues/2013/v3i4/papers/P aper-24.pdf

[32] Arul Kumaravelu, E.S.M. Suresh. Comparing the Higher
Education Quality Accreditation Parameters of India with Other Global Accreditation Agencies. Review of Research Journal, 8(8). 2013.http://oldror.lbp.world/ArticleDetails.as px?id=8485

[33] All India Survey on Higher Education (AISHE) Reports. http://aishe.nic.in/aishe/reports 